\title{
A Comprehensive Survey on Tools for Effective Alzheimer's Disease Detection
}

\author{
Vinutha, N., G.U. Vasanthakumar, P. Deepa Shenoy and K.R. Venugopal \\ Computer Science and Engineering, University Visvesvaraya College of Engineering, India
}

\begin{abstract}
Article history
Received: 16-06-2017

Revised: 16-08-2017

Accepted: 22-09-2017

Corresponding Author:

Vinutha, N.

Computer Science and

Engineering, University

Visvesvaraya College of

Engineering, India
\end{abstract}

Email: vinutha1v@gmail.com

\begin{abstract}
Neuroimaging is considered as a valuable technique to study the structure and function of the human brain. Rapid advancement in medical imaging technologies has contributed significantly towards the development of neuroimaging tools. These tools focus on extracting and enhancing the relevant information from brain images, which facilitates neuroimaging experts to make better and quick decision for diagnosing enormous number of patients without requiring manual interventions. This paper describes the general outline of such tools including image file formats, ability to handle data from multiple modalities, supported platforms, implemented language, advantages and disadvantages. This brief review of tools gives a clear outlook for researchers to utilize existing techniques to handle the image data obtained from different modalities and focus further for improving and developing advanced tools.
\end{abstract}

Keywords: Alzheimer's Disease, Computer Aided Diagnosis, Imaging, Morphometry, Neuroimaging Tools

\section{Introduction}

Alzheimer's Disease (AD) is one of the most common neurodegenerative diseases affecting older adults in their mid-60s (Joshi et al., 2010). Degeneration of the nervous system is mainly caused due to the deposition of amyloid plaques and neurofibrillary tangles (Hardy and Selkoe, 2002). Symptoms generally include memory loss, impairment in thinking, poor judgement and difficulty in recognising people. Since the number of patients with $\mathrm{AD}$ is expected to increase in near future, the National Institute of Aging has been focusing towards the development of effective techniques to diagnose $\mathrm{AD}$.

The transition to $\mathrm{AD}$ from normal aging is gradual; thus, many researchers have put attention towards the early detection of $\mathrm{AD}$. The early stage of $\mathrm{AD}$ is represented by the term, Mild Cognitive Impairment (MCI) (Petersen, 2011). Two different subtypes of MCI are Amnestic Mild Cognitive Impairment (aMCI) and Non amnestic Mild Cognitive Impairment (naMCI). aMCI patients have significantly impaired memory, but their cognitive functions remain effective. On the other hand, in case of naMCI, one or more cognitive skills are impaired but memory problem remains unaffected. Patients with MCI have increased risks of eventually developing $\mathrm{AD}$. Thus, identification of $\mathrm{AD}$ at the early stage is challenging and requires further investigations through popular neuroimaging techniques (Joshi et al., 2010).

Neuroimaging (Poldrack et al., 2008) or brain imaging is an important tool for understanding the anatomy and function of the human brain. Parameters such as tissue contraction, volume reduction and expansion, variation of shape, average intensity, metabolism rate and cortical thickness are of great interest to determine AD. These parameters can be obtained using a range of different techniques such as Magnetic Resonance Imaging (MRI), Fluorodeoxyglucose Positron Emission Tomography (FDG-PET), Diffusion Tensor Imaging (DTI), Functional Magnetic Resonance Imaging (fMRI), Computed Tomography (CT), Single Photon Emission Computed Tomography (SPECT) and Cerebrospinal Fluid (CSF) Biomarkers.

Among these techniques, MRI is reliable and noninvasive for producing high resolution images. It does not cause adverse sideeffects in patients; thus, it is considered as a significant technique to fetch both structural and functional information of the brain to study further. MRI is also preferred in predicting the shift from MCI to AD by either considering the whole brain or medial temporal lobe volume. However, MRI being a single modality technique does not provide comprehensive information. Hence, multimodal data analyzed by radiologists or neurologists using neuroimaging tools are preferred as sources of valuable 
information for assisting the diagnosis and prognosis of $\mathrm{AD}$ or $\mathrm{MCI}$ at early stages.

\section{Motivation}

Experts in the field of Neurology or Radiology are required to investigate problems associated with the complex brain structure. To overcome this manual and time consuming process, there is advancement towards the development of neuroimaging tools. Tools developed in the past are standalone techniques, which has motivated researchers and developers to design an open source tool with Graphical User Interface (GUI) providing compatibility to multiple operating systems. Based on the application and requirement, tools contributed by developers are enormous. Few tools provide easy and convenient way of installation and usage, but some of them have complicated procedures requiring high memory for processing three/four dimensional images to get desired results.

\section{Contribution}

In the present review, the ability of various neuroimaging tools to handle image data from different types of modalities, languages in which they are implemented, as well as various supporting functionalities are discussed.

\section{Organisation}

The paper is organized as follows: A brief overview of Alzheimer's Disease is provided in Section I. Related research are presented in Section II. Morphometry techniques are described in Section III. Tools in neuroimaging are explained in Section IV. A brief discussion about the tools are presented in Section V. A comprehensive conclusion is provided in Section VI.

\section{Literature Survey}

Many of open source tools have wide range of steps beginning with the preprocessing operation and ending with the analysis, which are essential to classify patients with neurodegenerative disorders. These steps are discussed in further paragraphs.

In the study of neurodegenerative disorders particularly Alzheimer's Disease (AD), image data are provided by the standard dataset like Alzheimer's Disease Neuroimaging Initiative (ADNI), Open Access Series of Imaging Studies (OASIS) and The Minimal Interval Resonance Imaging in Alzheimer's Disease (MIRIAD), which are available in DICOM (Digital Imaging and Communications in Medicine), NIFTI (Neuroimaging Informatics Technology Initiative), MINC and Analyze format. To suit the compatibility of data required for processing, the initial step is data conversion. It is required for converting images obtained from the dataset into a suitable format required for preprocessing. MRIcron, SPM and Free Surfer are few of the tools which are used for data conversion.

Sequences of preprocessing steps are followed according to the requirement of postprocessing operations desired for a particular application. It includes the removal of non-brain tissues (Zhuang et al., 2006) from T1 and T2 weighted Magnetic Resonance (MR) images. A skull-stripped subject image is spatially aligned to a standard template through the process of Spatial Normalisation (Friston et al., 1995), which helps to overcome the problem of brain shape variability across different subjects. AC-PC (Anterior CommissurePosterior Commissure) positioning aligns coordinates of the brain by their origin and axes, which is adopted as a convenient standard by the neuroimaging Community. Coordinate convertor converts the coordinate from display to Talairach and Montreal Neurological Institute (MNI) space. N3 inhomogeneity correction (Vovk et al., 2007) removes the intensity inhomogeneity across space caused during the acquisition of MR images from the scanner. Smoothening removes Gaussian Noise and Histogram Equalisation enhances images for redistributing the gray value to acquire an uniform distribution.

After preprocessing, the image is subjected to tissue segmentation (Tanabe et al., 1997; Vibha et al., 2007) to divide it into the gray matter, white matter and cerebrospinal fluid. Due to the neuronal loss caused by the deposition of amyloid plaques and neurofibrillary tangles, cortical gray matter reduction occurs to a greater extent compared to white matter and cerebrospinal fluid; hence, the gray matter is considered for further analysis. Segmentation (Kumar et al., 2009) is followed by registration to establish the correspondence between a set of images using transformation models and assess by considering similarity measures.

Image registration (Gholipour et al., 2007) is the most crucial step required in the medical imaging, which is described as the process of geometric alignment of the subject image to the template to bring it in correspondence. It involves the extraction of information from the feature space and transformation of the image required for matching. Affine, polynomial and elastic transformations are different types of spatial transformation techniques available to transform the image. Transformation technique is the fundamental characteristic in the process of registration to overlay images on each other. The amount of transformation is measured by the chosen similarity metric. Images considered during registration are taken from the same modality acquired during different time periods, which aid in determining the stage of the disease. In contrast, 
images taken from different modalities fuse multiple information, which result in improved analysis; moreover, images obtained from different views result in improved visualization. After aligning the image through registration, it is necessary to label brain regions to find the variability associated with each region.

Standard template or atlas is utilized to label anatomical regions of the brain (Hofman, 1988). The purpose of using a template is to provide a reference that includes a set of coordinates and associated anatomical labels. Demarcated labels on the template are then resampled through the warp transform to the subject MRI. This technique is consistent and less time consuming without any expertise needed to understand the involvement of different brain regions in the disease development. To further extract multiple features (Srinivasa et al., 2005) from different modalities, labeled structural image is mapped to the functional image of same subject through the process of coregistration. All extracted features are not informative; hence, the number of features is reduced by dimensionality reduction step to maximize the separation in feature space (Shenoy et al., 2005). Then, reduced features are used to perform accurate classifications of different stages of AD (Vibha et al., 2006).

\section{Morphometry}

Morphometry is a process of identifying local and global anatomical differences existing in the human brain. These morphological differences exist during the aging process and $\mathrm{AD}$ development; hence, it is considered as an effective biomarker for diagnosing neurodegenerative diseases. The main objective of morphometric techniques is to determine the one to one correspondence between images considered under study.

Voxel-Based Morphometry (VBM), Deformation Based Morphometry (DBM) or Tensor Based Morphometry (TBM), Feature Based Morphometry (FBM) and Shape Based Morphometry (SBM) are different types of morphometric techniques. Computation of morphometrics involves two common steps that are followed in all morphometric techniques. In the first step, subject image is geometrically aligned to the reference image and in the second step, statistics are computed for the region of interest or on whole brain. Many existing tools based on mathematical and statistical methods have been developed to process high resolution images automatically, leading to the characterization of differences exist between healthy individuals and patients.

Voxel-Based Morphometry (VBM) (Mechelli et al., 2005) uses a statistical method for accomplishing voxelwise comparison. VBM identifies differences in brain anatomy among a group of subjects. VBM features include increased or decreased tissue density and decreased gray matter and white matter volumes.
Extracted features are subjected to general linear modelling to obtain statistical maps. Then, statistical tests are performed using statistical maps to identify discriminative regions exist in a population.

Deformation Based Morphometry (DBM) (Savio and Graña, 2013) is a technique applied to identify macroscopic differences in brain shapes exist across the population. Thus, deformation fields are calculated by placing the brain to a standard template to find out positional differences between every voxel of individual brain and a standard brain. Finally, multivariate statistical methods are used to analyze a group of parameters derived from the whole brain.

Feature Based Morphometry (FBM) (Toews et al., 2010; Venugopal et al., 2009) aims to recognize structural differences exist only in a group of patients. FBM comprises four steps. First step begins with the extraction of features. In the second step, modelling of these features identifies the relationship between the distinctive feature and diseased brain. Statistical analysis is followed after the modeling to infer the information required to classify the subject.

Shape Based Morphometry (SBM) aims to identify local changes within the structure of interest. It begins with the preprocessing and normalization of the brain structure to a standard reference and geometrical variations are identified through the statistical analysis.

\section{Neuroimaging Tools}

Various neuroimaging tools that exist for processing brain images are explained in further sub sections. Advantages and disadvantages of these tools are discussed in Table 1 and important features supported by each tool are explained in Table 2.

\section{$S P M$}

Statistical Parametric Mapping (SPM) (Penny et al., 2001) is a licensed tool developed by the Wellcome Department of Imaging Neuroscience. SPM has matlab based functions and is available freely to the scientific community. SPM99, SPM2, SPM5, SPM8 and SPM12 are different versions of SPM. The older version supports the measurement of brain activity for PET (Positron Emission Tomography and fMRI (Functional Magnetic Resonance Imaging) modalities; however, the current version of SPM supports the analysis of SPECT, EEG (Electroencephalography) and MEG (Magnetoencephalogram) data. This tool has Digital Imaging and Communications in Medicine (DICOM) import option required for converting images from DICOM to Neuroimaging Informatics Technology Initiative (NIfTI) format, which is suitable for SPM and many other software packages. 
Table 1: Advatages and disadvantages of neuroimaging tools to detect Alzheimer's Disease

\begin{tabular}{|c|c|c|}
\hline Tools & Advantages & Disadvantages \\
\hline ABEAT & Provides parallel processing & Requirement of high computational time \\
\hline SPM & Supports to multiple plugins & Suitable for group analysis \\
\hline MARSBAR & Allows us to export ROI for use in other packages & Compatible only with SPM programs \\
\hline MRICRON & An excellent free-ware viewer of medical images & Limited display modes \\
\hline MIPAV & User friendly tool to analyse and visualise the image data & The execution of a program mainly depends on the hardware \\
\hline FSL & Interfaced well with other tools & Installation in windows requires virtual box \\
\hline HAMMER SUITE & Provides complete processing pipeline for MR images & Unable to access the current version \\
\hline DRAMMS & Well suited for deformable image registration & Memory consumption is high \\
\hline ITK-SNAP & Provides efficient and reliable segmentation & The tool has limited scope \\
\hline IMAGE J & Public domain open source software & Not supported to simultaneous statistical analysis of ROI's \\
\hline FREE SURFER & Robust tool for cortical surface analysis & Installation in windows requires virtual box \\
\hline 3D SLICER & An extensible tool & Requires more computational resources \\
\hline ANALYSE & $\begin{array}{l}\text { Comprehensive tool for studying } \\
\text { structural and functional relationship of brain image }\end{array}$ & Proprietary software \\
\hline SPHARM-PDM & Powerful tool for 3D shape analysis & Installation is difficult in linux \\
\hline pyMVPA & $\begin{array}{l}\text { A package for ease statistical analysis } \\
\text { with machine learning algorithms }\end{array}$ & Focus on advanced machine learning algorithms are necessary \\
\hline
\end{tabular}

Table 2: Neuroimaging tools to detect Alzheimer's disease

\begin{tabular}{|c|c|c|c|c|c|c|c|c|c|c|c|c|}
\hline Tools & $\begin{array}{l}\text { Coordinate } \\
\text { Convertor }\end{array}$ & $\begin{array}{l}\text { Data } \\
\text { Conversion }\end{array}$ & $\begin{array}{l}\text { Skull } \\
\text { Stripping }\end{array}$ & $\begin{array}{l}\text { Bias } \\
\text { Field } \\
\text { Correction }\end{array}$ & $\begin{array}{l}\text { Tissue } \\
\text { Segme- } \\
\text { ntation }\end{array}$ & $\begin{array}{l}\text { Regis- } \\
\text { tration }\end{array}$ & $\begin{array}{l}\text { Visuali- } \\
\text { sation }\end{array}$ & $\begin{array}{l}\text { Anal- } \\
\text { ysis }\end{array}$ & $\begin{array}{l}\text { Exten- } \\
\text { sibility }\end{array}$ & $\begin{array}{l}\text { Statistical } \\
\text { Navigation }\end{array}$ & $\begin{array}{l}\text { Image } \\
\text { Warping }\end{array}$ & Weblink \\
\hline $\begin{array}{l}\text { SPM } \\
\text { (Penny et al., 2001) }\end{array}$ & No & Yes & Yes & Yes & Yes & Yes & Yes & Yes & Yes & No & No & http://www.fil.ion.ucl.ac.uk/spm/software/spm12/ \\
\hline $\begin{array}{l}\text { MARSBAR } \\
\text { (Bahlmann } \text { et al., } 2015\end{array}$ & No & No & No & No & No & No & Yes & Yes & No & No & No & $\underline{\text { http://marsbar.sourceforge.net/ }}$ \\
\hline $\begin{array}{l}\text { WFU PICK ATLAS } \\
\text { (Callaghan } \text { et al., 2014) }\end{array}$ & Yes & No & No & No & No & No & Yes & Yes & No & No & No & $\underline{\text { http://fmri.wfubmc.edu/software/pickatlas }}$ \\
\hline $\begin{array}{l}\text { MRICRON } \\
\text { (Li et al., 2012) }\end{array}$ & No & Yes & No & No & No & No & Yes & Yes & No & No & No & http://neuro.debian.net/pkgs/mricron.html \\
\hline $\begin{array}{l}\text { MIPAV } \\
\text { (Bazin et al., 2007) }\end{array}$ & Yes & No & Yes & Yes & Yes & Yes & Yes & Yes & Yes & No & Yes & https://mipav.cit.nih.gov/pubwiki/index.php/ \\
\hline $\begin{array}{l}\text { FSL } \\
\text { (Smith et al., 2004) }\end{array}$ & No & Yes & Yes & Yes & Yes & Yes & Yes & Yes & Yes & No & No & https://fsl.fmrib.ox.ac.uk/fsl/fslwiki/ \\
\hline $\begin{array}{l}\text { HAMMER SUITE } \\
\text { (Wu et al., 2010) }\end{array}$ & No & No & Yes & Yes & Yes & Yes & Yes & No & No & No & Yes & $\underline{\text { https://www.med.unc.edu/bric/ideagroup/free-softwares/ }}$ \\
\hline $\begin{array}{l}\text { DRAMMS } \\
\text { (Ou et al., 2011) }\end{array}$ & No & No & No & No & No & Yes & Yes & No & Yes & No & Yes & https://www.cbica.upenn.edu/sbia/software/dramms/ \\
\hline $\begin{array}{l}\text { ABEAT } \\
\text { (Dai } \text { et al., 2013) }\end{array}$ & No & No & Yes & Yes & Yes & Yes & Yes & Yes & No & No & Yes & https://www.nitrc.org/projects/abeat/ \\
\hline $\begin{array}{l}\text { ANTS } \\
\text { (Avants et al., 2009) }\end{array}$ & No & No & No & No & No & Yes & Yes & Yes & No & No & No & http://stnava.github.io/ANTs/ \\
\hline $\begin{array}{l}\text { ITK-SNAP } \\
\text { (Robb, 2001) }\end{array}$ & No & No & No & No & No & No & Yes & Yes & Yes & Yes & Ye & http://www.itksnap.org/pmwiki/ \\
\hline $\begin{array}{l}\text { BRAIN SUITE } \\
\text { (Shattuck and } \\
\text { Leahy, 2002) }\end{array}$ & No & No & Yes & No & No & Yes & Yes & No & No & No & Yes & http://brainsuite.org/ \\
\hline $\begin{array}{l}\text { BIOIMAGE SUITE } \\
\text { (Papademetris } \text { et al., 2006) }\end{array}$ & No & No & Yes & Yes & Yes & Yes & Yes & Yes & Yes & No & No & $\underline{\text { http://bioimagesuite.yale.edu/ }}$ \\
\hline $\begin{array}{l}\text { 3D SLICER } \\
\text { (Fedorov } \text { et al., 2012) }\end{array}$ & No & Yes & Yes & Yes & Yes & Yes & Yes & Yes & Yes & No & No & https://www.slicer.org/ \\
\hline $\begin{array}{l}\text { FREE SURFER } \\
\text { (Fischl, 2012) }\end{array}$ & No & Yes & Yes & No & No & Yes & Yes & Yes & No & No & No & http://freesurfer.net/ \\
\hline $\begin{array}{l}\text { BRAIN VOYAGER } \\
\text { (Goebel and Jansma, 2004) }\end{array}$ & No & No & No & No & No & Yes & Yes & Yes & No & Yes & No & http://www.brainvoyager.com/ \\
\hline
\end{tabular}

SPM performs various preprocessing operations like realignment, motion correction, spatial normalization and removal of Gaussian noise. Realignment and motion correction adjusts the image in such a way that each voxel corresponds to a same site in the brain. The process of spatial normalization allows each subject image to be translated, rotated, scaled and warped according to the standard template. After preprocessing, significant functionality of SPM is to determine the morphometry at each voxel by general linear modeling technique. The morphometry is analyzed by statistical models such as t-test, ANOVA, MANCOVA, ANCOVA, simple regression, linear regression, multiple regression and multivariate regression to perform comparisons among a group of subjects.

\section{MARSBAR}

MARSBAR (MARSeille Boite A Region d Interest) (Bahlmann et al., 2015) is a plug-in of SPM, which is compatible to SPM 99, 2, 5 and 8 version. It is well suited for functional Region of Interest (ROI) analysis using PET and MRI modalities. MARSBAR provides a view option which displays ROI in a structural image and allows exporting of images to use in other packages. The interface given by MARSBAR defines ROI using shapes, activation cluster and binary images. It allows importing SPM activations and cluster images obtained by SPM result interface as ROIs. The data extraction for regions can also be done without SPM preprocessing. Finally, SPM statistics machinery helps to interpret and analyze ROI data. 


\section{WFU Pick Atlas}

WFU (Wake Forest University) Pick Atlas (Callaghan et al., 2014) is a plug-in of SPM, which is compatible to SPM99, SPM2, SPM5 and SPM12 versions and performs atlas based ROI analysis. It supports NIfTI file format and provides coordinate converter that converts coordinates from display to Talairach and MNI space. It uses Talairach Daemon database to generate ROI mask. It provides an option to access available atlases and also incorporates additional atlases. The option of look up file available in WFU, lists atlases to be loaded along with their corresponding volumes. It is also possible to create user defined shapes by defining centre, radius and dimensions.

\section{MRICRON}

MRICRON (Li et al., 2012) is the newer version of MRIcro developed by Chris Rorden. It is a GUI (Graphical User Interface) based tool applied in the visualization and analysis of functional and structural images like MRI, PET and fMRI. It is written in C language and suitable for Windows, Linux and Mac OS $\mathrm{X}$ platform. NIfTI and Analyze are image formats supported for this tool. Feature available in this software is image conversion, to obtain 3D anatomical region of interest with computed volume and intensity. There is an option to change X (Left/Right), Y (Anterior/Posterior) and $\mathrm{Z}$ (Superior/ Inferior) values, which in turn changes the axial, sagittal and coronal views of the displayed image. It has other options such as zoom to enlarge the view of the brain, multislice window option to show a series of slices of selected volume, as well as option to perform voxel wise statistical analysis. Images are exported to other platforms by creating Analyze format header.

\section{MIPAV}

MIPAV (Medical Image Processing Analysis and Visualization) (Bazin et al., 2007) is a freely available medical image processing software, which is written in JAVA language and used for quantitative neuroimaging analysis of MRI, PET and CT data. It is developed by the National Institutes of Health and is compatible to Windows, Linux and Mac OS X platforms. NIFTI, AFNI, MINC, DICOM and TIFF are supported image formats suitable for processing. Preprocessing operations provided by MIPAV are semi-automatic brain extraction and Talairach alignment of an image to a standardized coordinate system. It also provides an option of loading Talairach atlas of a particular Volume of Interest (VOI) onto the aligned brain region and helps to calculate the volume and mean intensity using VOI analysis tool.

\section{FSL}

FSL (FMRIB Software Library) (Smith et al., 2004) is a software library implemented in $\mathrm{C}++$ language and developed by FMRIB (Functional Magnetic Resonance Imaging of the Brain) analysis group. It comprises image analysis and statistical tool for processing structural, functional and diffusion MRI. FSL is operated either by GUI (Grahical User Interface) or command line and is compatible to Linux, Apple OS X, Centos Linux, Redhat Linux and Windows operating system. Features supported by FSL are as follows: conversion to Analyze and NIfTI formats with the help of command line utilities; skull-stripping using BET (Brain Extraction Tool); tissue segmentation by FAST (FMRIB's Automated Segmentation Tool); affine registration using FLIRT (FMRIB's Linear Image Registration Tool); subcortical structure segmentation by incorporating prior anatomical information through the explicit shape model followed by vertex analysis and volumetric analysis; nonlinear noise reduction by SUSAN (Smallest Univalue Segment Assimilating Nucleus); gray matter density analysis by FSLVBM (Voxel Based Morphometry); SIENAX dependent estimation of the cross sectional atrophy at a single time point (atrophy state) and SIENA dependent determination of the longitudinal atrophy of the cortical gray matter between two time points (atrophy rate) and visualization of 2D and 3D data.

\section{HAMMER SUITE}

HAMMER SUITE (Hierarchal Attribute Matching Mechanism for Elastic Registration) (Wu et al., 2010) is an elastic registration procedure for high dimensional warping of brain images developed by the Section of Biomedical Image Analysis (SBIA) at the University of Pennsylvania. This tool is available for both Windows and Linux platforms and it is designed to process only Analyze image format of brain images. Longitudinal and cross sectional brain images are preprocessed using following steps: reorientation, alignment, skull-stripping, deformable registration and tissue segmentation. It provides models for normalization, labeling anatomical regions of interest and also to perform label correction. After preprocessing and labeling, RAVENS MAP (Tissue Density Maps) is computed for each subject brain to perform voxel based analysis on regional volumetrics. Group analysis is performed by applying statistical methods on to RAVENS MAP to examine disease effects, correlation with clinical measurements and regional volumetrics.

\section{DRAMMS}

DRAMMS (Deformable Registration via Attribute Matching and Mutual Saliency Weighting) (Ou et al., 2011) is suitable for linux and Mac OS X operating systems. Prior 
knowledge, presegmentation, or human intervention is not required for subjecting the brain into DRAMMS. Features of DRAMMS include cross subject registration of the same organ among different patients, mono and multimodality registration for MRI-MRI, MRI-PET and PET-PET images, longitudinal registration of images for different time series to examine changes of brain during a given time period and warping of template to the subject brain. It also has an option to calculate Jacobian Determinant Maps to find the voxel displacement of an entire image and RAVENS MAPS to determine changes with the tissue density.

\section{aBEAT}

aBEAT (Adult Brain Extraction and Analysis Toolbox) (Dai et al., 2013) is a graphical user interface implemented in matlab and $\mathrm{C}++$ and developed by IDEA group, which is compatible with Linux operating system. Only T1 weighted longitudinal and cross sectional images with analyze file format are supported by the processing operation available in the toolbox. It performs image preprocessing such as reorientation, re-sampling in accordance with template images, N3 correction, histogram matching, skull-stripping and tissue segmentation. It also performs brain volume labeling and volume measurements of different brain regions.

\section{ANTS}

ANTS (Advanced Normalization Tools) (Avants et al. 2009) package provides advanced tools for the brain and image mapping. It is developed by BRAIN AVANTS and supported by PICSL. It is open source software supported to run on Linux and Macintosh platforms. Tool performs well across species and for different organ system. Data formats suitable for processing are NIfTI, Analyze and Metaimage. Features of ANTS include N3/N4 bias correction, warping, registration using diffeomorphic and deformation based transformation, tissue based image segmentation, prior based image segmentation, cortical thickness measurement, Laplacian based cortical thickness estimation, diffeomorphic registration based cortical thickness (DiReCT) estimation and visualization of biomedical images.

\section{ITK-SNAP}

ITK-SNAP (Insight Toolkit Snake Automatic Partitioning Tool) (Gao et al., 2012) is open source software developed and supported by the National Institute of Biomedical Imaging and Bioengineering (NIBIB), which is compatible to Linux, Mac OS and Windows platforms. NIfTI, DICOM and other 3D image formats are supported for processing. Features provided by ITK-SNAP include cross hair and zoom tool for image navigation, manual delineation, affine alignment, rigid alignment, quick loading of the series of DICOM images in the same session and semi-automatic segmentation using active contour methods. Options of loading and saving 3D segmented volumes of 3D images are available. It also provides manual and semiautomatic labeling of anatomical structures and lesions in 3D images and estimation of the volume and statistics of these labeled regions.

\section{ANALYSE}

ANALYZE (Robb, 2001) is a visualization and analysis software package used in medical imaging. It is developed by Biomedical Imaging Resource (BIR) at Mayo clinic. It is a commercial program suitable for multimodal images like MRI, CT, PET and SPECT. Functionality of Analyze are cropping, resizing, rotation, image filtering, image enhancement, skull-stripping, interactive volume segmentation, morphological operations, image alignment, nonlinear registration, region and volume definition, statistical analysis, advanced visualization and volume rendering operations.

\section{MNI DISPLAY}

MNI DISPLAY (Kabani et al., 2001) is developed by David Mac Donald at McConnell Brain Imaging Centre and used for the visualization of DTI, fMRI and PET modalities. Linux and Mac OS X are operating systems supported by this tool. Visualization features of MNI Display include displaying intensity crosssections along $\mathrm{X}, \mathrm{Y}, \mathrm{Z}$, or time axes, overlaying multiple volumetric images of different sample grid sizes, visualization of 3D surfaces and intersection of 3D surfaces with the volumetric data. Segmentation features of MNI Display are per-voxel labeling of the volumetric data and annotation of structural features on either a surface or a volumetric dataset. It also provides morphological operations like powerful fill, dilation and erosion.

\section{IMAGEJ}

IMAGEJ (Schindelin et al., 2012) is open source software developed at the National Institutes of Health (NIH) for the image processing and analysis of biological and medical data. It is available freely to the public domain and is compatible to Windows, Linux and Mac OS $\mathrm{X}$ platforms. Image J is developed in Java language; hence, it provides extensibility to additional plugins. DICOM, NIfTI, Analyze and JPEG are image file formats supported by this tool. Image processing functions of ImageJ include median filtering, sharpening, smoothening, scaling, rotation, edge detection, contrast manipulation, labeling, VOI measurements and visualization.

\section{BRAIN SUITE}

BRAIN SUITE (Shattuck et al., 2002) is an image analysis tool used for processing MRI images of human head. It is implemented in $\mathrm{C}++$ language and developed 
by David Shattuck of Ahmanson-Lovelace Brain Mapping Center at UCLA and Richard Leahy of Biomedical Imaging Group at USC. It provides GUI for Windows and Mac OS X and command line interface for Linux operating system. BRAIN SUITE supported features are brain surface extraction, bias field correction, 3D alignment, surface smoothening, histogram computation, tissue classification, topological correction, cerebrum labeling, extracting cortical surface mesh models, registering and labeling the surface and volume of the brain, defining the anatomical region of interest through the automated registration of subject image to the labeled atlas and mapping of multiple images to common atlas space. It also contains tool for visualizing and exploring MRI data, diffusion data and regional connectivity.

\section{BIOIMAGE SUITE}

BIOIMAGE SUITE (Papademetris et al., 2006) is the software developed by Xenophon Papademetris at the Yale University School of Medicine and it consists of a collection of image analysis programs written in $\mathrm{C}++$ language. It provides both graphical and command line Interface for interactive and batch mode processing. It is suitable for Windows, Linux and Mac OS X platforms. FSL is easily integrated with this tool, which aids in performing skullstripping and tissue classification. In addition to FSL, it is easily integrated to other packages such as WFU PICK ATLAS, SPM and AFNI tool. Features provided by BIOIMAGE SUITE are image import, understanding the basic information of image, image smoothening/filtering, re-slicing, reorientation, bias field correction, cropping, rigid and affine registration methods to align serial MRI and multimodal data, warping of single subject data to a common reference space, deformable surface segmentation, as well as different methods of voxel classification such as simple histogram, single channel markov random field and exponential-fit method. It also provides orthogonal and mosaic views for displaying images.

\section{$3 D$ SLICER}

3D SLICER (Fedorov et al., 2012) is an open source software tool developed collaboratively by Surgical Planning Laboratory at Brigham and Women's Hospital at the MIT AI LAB and it shows more attention towards visualization and analysis of the medical data. It is implemented in $\mathrm{C}++$ and it is available to windows, Red Hat Linux, Mac OS and IRIX platforms. Various modalities of images handled by this tool are MRI, CT, Ultrasound and Nuclear Medicine in DICOM, NIfTI, Analyze and META data formats. It provides option for the integration with multiple toolkits due to its extensibility and flexibility. Functions of 3D SLICER are format conversion, de-noising, nonlinear/anisotropic smoothening, mapping, multimodal registration, rigid registration, affine registration, B-spline registration, atlas based segmentation, region growing segmentation, quantification of image through volume measurements and interactive visualization of volumetric meshes, polygonal meshes and volume renderings.

\section{FREE SURFER}

FREE SURFER (Fischl, 2012) is an open source brain imaging software developed by Martinos center for Biomedical Imaging and is applied in analyzing structural MRI scan in NIfTI format. This tool is compatible to Linux, Mac OS and Windows platforms and is implemented in $\mathrm{C}++$ language. Tkmedit and tksurfer tools are used for visualizing and editing images. Functions supported by FREE SURFER include conversion of the subject scan to a standard NIfTI format, orientation of images according to a standard template, skull-stripping, image registration, automated cortical segmentation, subcortical segmentation, cortical parcellation, labeling of subject scans using pre-existing labels, brain morphometry and fMRI analysis. Statistical measures estimated for subcortical segmented structures are number of voxels, volume and mean intensity. Different statistical measures provided for cortical parcellated brain scan are number of vertices, surface area, gray matter volume, average thickness, folding index and intrinsic curvature index.

\section{SPHARM-PDM}

SPHARM-PDM (Spherical Harmonics-Point Distribution Model) (AlHadidi et al., 2012) is a statistical shape analysis tool written in $\mathrm{C} / \mathrm{C}++$ language and is developed by the Neuroimage Research and Analysis Laboratories. Linux, Mac OS and Windows are suitable platforms to SPHARM-PDM Toolbox. It is available as an external extension to 3D Slicer. Features of SPHARM-PDM include preprocessing of 3D binary image data such as removal of bad edge connectivity, bad vertex connectivity and 3D holes, which are carried out to create spherical topology. The uniform mapping of an object surface to the surface of unit sphere with minimized angle distortion is performed through SPHARM parameterization. SPHARM alignment creates a shape descriptor from the normalized SPHARM coefficients and it is used for the group analysis. Principal component analysis and t-test are available for statistical shape analysis.

\section{pyMVPA}

pyMVPA (Multivariate Pattern Analysis in python) (Hanke et al., 2009) is a freely available software written in python language and it is used for statistical analysis of large dataset in neuroimaging. It is developed by Michael Hanke to provide an easy installation and integration to other related software packages and 
libraries consisting of machine learning algorithms (Joshi et al., 2010). pyMVPA is suitable for windows and Mac OS platforms. NIfTI and Analyze are supported file formats for the processing. It has built-in functions for feature selection such as simple thresholding, incremental feature search and recursive feature selection. Algorithms available for the dimensionality reduction are principal component analysis and independent component analysis. Different classifiers provided are K-Nearest Neighbor, ridge regression, support vector machine, sparse multinomial logistic regression and penalized logistic regression. Different methods used for the computation of statistics are binomial test and permutation test.

\section{AIR}

AIR (Automated Image Registration) (Woods et al., 1998) is a software package used for the automated registration of $3 \mathrm{D}$ and $2 \mathrm{D}$ brain images. It is free licensed tool implemented in $\mathrm{C}$ language and compatible with Windows, UNIX and Macintosh platforms. The tool provides features such as intrasubject-intramodality registration (MRI-MRI, PET-PET), intrasubjectintermodality registration (MRI-PET) and intersubject registration (Subject-Subject or Subject-Atlas Template).

\section{BRAIN VOYAGER}

BRAIN VOYAGER (Goebel and Jansma, 2004) is a multimodal analysis tool used for the analysis of functional and structural MRI, Electroencephalography (EEG), DTI and MEG (Magnetoencephalography) data. It is suitable for Windows, Linux and Mac OS $\mathrm{X}$ platforms. Tool performs automatic co-registration of the functional and diffusion weighted data with $3 \mathrm{D}$ anatomical dataset and also supports advanced visualization of the anatomical and functional data set. Preprocessing operations available in BRAIN VOYAGER are motion correction, high pass filtering and slice scan time correction and cortex based inter subject alignment based on gyral and sulcal pattern. Both the individual and group analysis of brain images is performed by volume and cortex based analyses using independent component analysis. Multi-subject volume of interest and surface patch of interest analyses are also supported. In addition, multivoxel pattern analysis is performed through support Vector machine and recursive feature elimination.

\section{Discussion}

Contribution of various image modalities has increased the quantity and quality of neuroimaging data tremendously. It is necessary to use computationally intensive programs to transform the image data into a suitable form, which is required for the analysis and interpretation of brain behavior. There is a vast choice of user friendly and freely available neuroimaging tools; however, they are limited to perform only specific tasks. To overcome this limitation, frameworks are designed using advanced and effective software packages. The integration of software packages creates a standardized and single unified environment to process images using multiple steps and extract required features suitable for the analysis. Some of software packages such as FSL, 3D SLICER, FREESURFER and MIPAV are suitable to develop user friendly processing pipeline as they are expandable and can be interfaced well with other programs.

\section{Conclusion}

A comprehensive discussion about numerous tools in this paper gives a clear idea to the beginners to decide a specific tool for handling the image data obtained from the human brain. This will help researchers to utilize existing functionalities of these tools, interpret complex data and focus towards further enhancements by extracting features suitable for the analysis. Existing tools lack flexibility and are supported only by computers with specific hardware requirements such as high memory and high processing capacity. Thus, the development of tools is required to optimize the memory consumption, processing overheads and reduce the computational time. Further, there are rapid advancements towards imaging technologies leading to the acquisition of multimodal images from hybrid scanners. Therefore, advanced tools require parallel computing methods and extensibility and have to be interfaced well with other software packages.

\section{Author's Contribution}

All authors were involved in manuscript preparation.

\section{Ethics}

This article is original and contains unpublished material. The corresponding author confirms that all of the other authors have read and approved the manuscript and there are no ethical issues involved.

\section{References}

AlHadidi, A., L.H. Cevidanes, B. Paniagua, R. Cook and F. Festy et al., 2012. 3D quantification of mandibular asymmetry using the SPHARM-PDM tool box. Int. J. Comput. Assisted Radiology Surgery, 7: 265-271.

DOI: $10.1007 / \mathrm{s} 11548-011-0665-2$

Avants, B.B., N. Tustison and G. Song, 2009. Advanced Normalization Tools (ANTS). Insight J., 2: 1-5. 
Bahlmann, J., E. Aarts and M. D’Esposito, 2015. Influence of motivation on control hierarchy in the human frontal cortex. J. Neurosci., 35: 3207-3217. DOI: 10.1523/JNEUROSCI.2389-14.2015

Bazin, P.L., J.L. Cuzzocreo, M.A. Yassa, W. Gandler and M.J. McAuliffe et al., 2007. Volumetric neuroimage analysis extensions for the MIPAV software package. J. Neurosci. Methods, 165: 111-121.

Callaghan, M.F., P. Freund, B. Draganski, E. Anderson and M. Cappelletti et al., 2014. Widespread age-related differences in the human brain microstructure revealed by quantitative magnetic resonance imaging. Neurobiol. Aging, 35: 1862-1872. DOI: 10.1016/j.neurobiolaging.2014.02.008

Dai, Y., Y. Wang, L. Wang, G. Wu and F. Shi et al., 2013. aBEAT: A toolbox for consistent analysis of longitudinal adult brain MRI. PloS One, 8: e60344. DOI: 10.1371/journal.pone.0060344

Fedorov, A., R. Beichel, J. Kalpathy-Cramer, J. Finet and J.C. Fillion-Robin et al., 2012. 3D slicer as an image computing platform for the quantitative imaging network. Magnetic Resonance Imaging, 30: 1323-1341. DOI: 10.1016/j.mri.2012.05.001

Fischl, B., 2012. Freesurfer. Neuroimage, 62: 774- 781. DOI: 10.1016/j.neuroimage.2012.01.021

Friston, K., J. Ashburner, C.D. Frith, J.B. Poline and J.D. Heather et al., 1995. Spatial registration and normalization of images. Human Brain Mapping, 3: 165-189. DOI: $10.1002 / \mathrm{hbm} .460030303$

Gao, Y., R. Kikinis, S. Bouix, M. Shenton and A. Tannenbaum, 2012. A 3D interactive multi-object segmentation tool using local robust statistics driven active contours. Med. Image Anal., 16: 1216-1227.

Gholipour, A., N. Kehtarnavaz, R. Briggs, M. Devous and K. Gopinath, 2007. Brain functional localization: A survey of image registration techniques. IEEE Trans. Medical Imaging, 26: 427-451. DOI: 10.1109/TMI.2007.892508

Goebel, R. and H. Jansma, 2004. Brain Voyager QX. Psychology Software Tools: Pittsburgh, PA.

Hanke, M., Y.O. Halchenko, P.B. Sederberg, S.J. Hanson and J.V. Haxby et al., 2009. Pymvpa: A python toolbox for multivariate pattern analysis of functional MRI data. Neuroinformatics, 7: 37-53. DOI: $10.1007 / \mathrm{s} 12021-008-9041-y$

Hardy, J. and D.J. Selkoe, 2002. The amyloid hypothesis of Alzheimer's disease: Progress and problems on the road to therapeutics. Science, 297: 353-356. DOI: $10.1126 /$ science. 1072994

Hofman, M., 1988. Size and shape of the cerebral cortex in mammals. Brain Behav. Evolution, 32: 17-26. DOI: $10.1159 / 000116529$

Joshi, S., P.D. Shenoy, K.R. Venugopal and L.M. Patnaik, 2010. Classification of neurodegenerative disorders based on major risk factors employing machine learning techniques. Int. J. Eng. Technol., 2: 350-356. DOI: 10.7763/IJET.2010.V2.146
Joshi, S., P.D. Shenoy, P. L. Rrashmi, K.R. Venugopal and L.M. Patnaik, 2010. Classification of Alzheimer's Disease and Parkinson's Disease by using machine learning and neural network methods. Proceedings of the 2nd International Conference on Machine Learning and Computing, Feb. 9-11, IEEE Xplore press, pp: 218-222, 2010. Bangalore, India. DOI: 10.1109/ICMLC.2010.45

Joshi, S., V. Simha, P.D. Shenoy, K.R. Venugopal and L.M. Patnaik, 2010. Classification and treatment of different stages of Alzheimer's disease using various machine learning methods. Int. J. Bioinformatics Res., 2: 44-52.

Kabani, N., G. Le Goualher, D. MacDonald and A.C. Evans, 2001. Measurement of cortical thickness using an automated 3-D algorithm: A validation study. Neuroimage, 13: 375-380.

DOI: 10.1006/nimg.2000.0652

Kumar, H.C.S., K. Raja, K.R. Venugopal and L.M. Patnaik, 2009. Automatic image segmentation using wavelets. Int. J. Computer Sci. Network Security, 9: 305-313.

Li, J., P. Pan, R. Huang and H. Shang, 2012. A metaanalysis of voxel-based morphometry studies of white matter volume alterations in Alzheimer's Disease. Neurosci. Biobehav. Rev., 36: 757-763.

Mechelli, A., C.J. Price, K.J. Friston and J. Ashburner, 2005. Voxel-based morphometry of the human brain: Methods and applications. Current Medical Imaging Rev., 1: 105-113. DOI: $10.2174 / 1573405054038726$

Ou, Y., A. Sotiras, N. Paragios and C. Davatzikos, 2011. DRAMMS: Deformable registration via attribute matching and mutual-saliency weighting. Medical Image Anal., 15: 622-639.

DOI: $10.1016 /$ j.media.2010.07.002

Papademetris, X., M.P. Jackowski, N. Rajeevan, M. DiStasio and H. Okuda et al., 2006. BioImage Suite: An integrated medical image analysis suite: An update. Insight J.

Penny, W.D., K.J. Friston, J.T. Ashburner, S.J. Kiebel and T.E. Nichols, 2001. Statistical parametric mapping: The analysis of functional brain images.

Petersen, R.C., 2011. Mild cognitive impairment. New England J. Med., 364: 2227-2234.

Poldrack, R.A., A.D. Wagner, J.T. Cacioppo, G.G. Berntson and H.C. Nusbaum, 2008. Neuroimaging as a new tool in the toolbox of psychological science. Current Directions Psychological Sci., 17: 62-67. DOI: 10.1111/j.1467-8721.2008.00550.x

Robb, R.A., 2001. The biomedical imaging resource at mayo clinic. IEEE Trans. Medical Imaging, 20: 854-867. 
Savio, A. and M. Graña, 2013. Deformation based feature selection for computer aided diagnosis of Alzheimer's disease. Expert Systems Applic., 40: 1619-1628. DOI: 10.1016/j.eswa.2012.09.009

Schindelin, J., I. Arganda-Carreras, E. Frise, V. Kaynig and M. Longair et al., 2012. Fiji: An open-source platform for biologicalimage analysis. Nature Methods, 9: 676-682. DOI: 10.1038/nmeth.2019

Shattuck, D.W. and R.M. Leahy, 2002. BrainSuite: An automated cortical surface identification tool. Med. Image Anal., 6: 129-142.

DOI: $10.1016 / \mathrm{S} 1361-8415(02) 00054-3$

Shenoy, P.D., K.G. Srinivasa, K.R. Venugopal and L.M. Patnaik, 2005. Dynamic association rule mining using genetic algorithms. Int. J. Intelligent Data Anal., 9: 439-453.

Smith, S.M., M. Jenkinson, M.W. Woolrich, C.F. Beckmann and T.E. Behrens et al., 2004. Advances in functional and structural MR image analysis and implementation as FSL. Neuroimage, 23: S208S219. DOI: 10.1016/j.neuroimage.2004.07.051

Srinivasa, K.G., A. Singh, A.O. Thomas, K.R. Venugopal and L.M. Patnaik, 2005. Generic feature extraction for classification using fuzzy c-means clustering. Proceedings of the 3rd International Conference on Intelligent Sensing and Information Processing, Dec. 14-17, IEEE Xplore press, Bangalore, India, pp: 33-38.

DOI: 10.1109/ICISIP.2005.1619409

Tanabe, J.L., D. Amend, N. Schuff, V. DiSclafani and F. Ezekiel et al., 1997. Tissue segmentation of the brain in Alzheimer disease. Am. J. Neuroradiology, 18: $115-123$.

Toews, M., W. Wells, D.L. Collins and T. Arbel, 2010. Feature-based morphometry: Discovering grouprelated anatomical patterns. NeuroImage, 49: 2318 2327. DOI: 10.1016/j.neuroimage.2009.10.032
Venugopal, K.R., K.G. Srinivasa and L.M. Patnaik, 2009. Soft Computing for Data Mining Applications. Springer.

Vibha, L., G.M. Harshavardhan, K. Pranaw, P.D. Shenoy and K.R. Venugopal et al., 2006. Classification of mammograms using decision trees. Proceedings of the 10th International Database Engineering and Applications Symposium, Dec. 1114, IEEE Xplore press, Delhi, India, pp: 263-266. DOI: $10.1109 /$ IDEAS.2006.14

Vibha, L., G.M. Harshavardhan, K. Pranaw, P.D. Shenoy and K.R. Venugopal et al., 2007. Lesion detection using segmentation and classification of mammograms. Proceedings of the 25th International Multi-Conference: Artificial Intelligence and Applications, Feb. 12-14, ACTA Press Anaheim, Innsbruck, Austria, pp: 311-316.

Vovk, U., F. Pernus and B. Likar, 2007. A review of methods for correction of intensity inhomogeneity in MRI. IEEE Transactions Medical Imaging, 26: $405-$ 421. DOI: $10.1109 /$ TMI.2006.891486

Woods, R.P., S.T. Grafton, C.J. Holmes, S.R. Cherry and J.C. Mazziotta, 1998. Automated image registration: I. general methods and intrasubject, intramodality validation. J. Comput. Assisted Tomography, 22: 139-152 DOI: 10.1097/00004728-199801000-00027

Wu, G., P.T. Yap, M. Kim and D. Shen, 2010. TPSHAMMER: Improving HAMMER registration algorithm by soft correspondence matching and thin-plate splines based deformation interpolation. NeuroImage, 49: 2225-2233.

DOI: 10.1016/j.neuroimage.2009.10.065

Zhuang, A.H., D.J. Valentino and A.W. Toga, 2006. Skullstripping magnetic resonance brain images using a model-based level set. NeuroImage, 32: 79-92. DOI: 10.1016/j.neuroimage.2006.03.019 